\title{
On Hall magnetohydrodynamics equilibria
}

\author{
G. N. Throumoulopoulos \\ University of Ioannina, Association Euratom - Hellenic Republic, \\ Section of Theoretical Physics, GR 45110 Ioannina, Greece \\ H. Tasso \\ Max-Planck-Institut für Plasmaphysik, Euratom Association, \\ D-85748 Garching, Germany
}

\begin{abstract}
Steady states are studied in the framework of ideal Hall magnetohydrodynamics (HMHD) model in arbitrary and axisymmetric geometries. In arbitrary geometry, conditions are found under which certain magnetohydrodynamics (MHD) equilibrium solutions can also satisfy the HMHD equations. For axisymmetric plasmas reduced equations are derived for uniform electron temperatures on magnetic surfaces and either barotropic ions or incompressible ion flows. The Hall and electron pressure gradient terms result in a deviation of the magnetic from the ion velocity surfaces and consequently the axisymmetric equilibria obey a set of coupled partial differential equations, one for the poloidal magnetic flux function and the other for a flux function labeling the ion velocity surfaces. Furthermore, the characteristics of certain classes of axisymmetric steady states with side conditions, as flows parallel to the magnetic field or purely poloidal incompressible flows, are identified and compared with respective MHD equilibria. Unlike in the frame of MHD, steady states with parallel axisymetric flows must be incompressible and equilibria with purely poloidal incompressible flows are possible. Certain analytic axisymmetric solutions are also constructed.
\end{abstract}




\section{Introduction}

Hall magnetohydrodynamics (HMHD) is a simple two-fluid model in the approximation of very small electron mass. Consequently the electron momentum equation for infinite electrical conductivity can be put in the Ohm's law form

$$
\mathbf{E}+\mathbf{v}_{i} \times \mathbf{B}=\frac{\mathbf{j} \times \mathbf{B}}{e n}-\frac{\nabla P_{e}}{e n},
$$

where $\mathbf{v}_{i}$ is the ion fluid element velocity. The right hand side (rhs) of (1) contains the Hall and electron pressure gradient terms. As concerns equilibrium studies the (single-fluid) ideal magnetohydrodynamics (MHD) model has usually been employed in which the rhs of (1) is neglected. There are, however, low density astrophysical plasmas in which the Hall and $\boldsymbol{\nabla} P_{e}$ terms become comparable with the other terms. Also, large electron pressure gradients are developed in the improved confinement regimes of laboratory fusion plasmas in connection with internal and edge transport barriers. In addition, in the edge region the density is up to four orders of magnitude lower than in plasma core.

Over the last years there is an increasing number of papers on HMHD equilibria. In Ref. [1] HMHD axisymmetric isentropic equilibria with toroidal rotation were investigated as deviations from the MHD ones. In that paper the $\boldsymbol{\nabla} P_{e}$ term is neglected. The so called Double-Beltrami HMHD steady states were found in Ref. [6] and then were employed to study discontinuous changes of the equilibrium configurations with applications to solar phenomena [3] and to discuss the role of the singular perturbation due to the Hall effect in producing multi-scale structures [4]. Ref. [5] is devoted to axisymmetric HMHD equilibria for a barotropic ion equation of state and vanishing ion velocity. A variational principle in connection with a constrained self-organization process was proposed in Ref. [6] to construct Hall-MHD relaxed states. Helically symmetric Hall-MHD stationary states were studied recently in Ref. [7]. Also, there are certain publications on axisymmetric equilibria in the framework of the full two-fluid model [8]-[12].

In the present report we study ideal HMHD equilibria by including the convective ion velocity term, $\left(\mathbf{v}_{i} \cdot \boldsymbol{\nabla}\right) \mathbf{v}_{i}$, in the momentum equation together with the Hall and $\boldsymbol{\nabla} P_{e}$ terms in Ohm's law. Unlike in the case of MHD, the latter two terms in general make the ion velocity surfaces to depart from the magnetic surfaces. This in conjunction with the $\left(\mathbf{v}_{i} \cdot \boldsymbol{\nabla}\right) \mathbf{v}_{i}$ term makes a 
self-consistent treatment of the problem apparently more complicated than in MHD.

The work is organized as follows. First we examine under which conditions MHD solutions in arbitrary geometry can be extended to the HMHD model. This is the subject of Sec. II. Specifically, particular stationary steady states $\left[\left(\mathbf{v}_{i} \cdot \boldsymbol{\nabla}\right) \mathbf{v}_{i} \neq 0\right]$ with incompressible flows parallel to $\mathbf{B}$ are considered in addition to general quasi-static equilibria $\left[\left(\mathbf{v}_{i} \cdot \boldsymbol{\nabla}\right) \mathbf{v}_{i}=0\right]$. In Section III reduced stationary axisymmetric equations are derived for uniform electron temperatures on magnetic surfaces and either barotropic ion equation of state or incompressible ion flows. Certain classes of axisymmetric side-condition equilibria are then examined in Sec. IV, i.e. equilibria with constant density, flows parallel to $\mathbf{B}$, Beltrami-like flows, toroidal barotropic flows and poloidal incompressible flows. Certain general characteristics of these equilibria are identified and compared with the respective MHD ones. Section V summarizes the conclusions.

\section{Certain three-dimensional equilibria}

The HMHD equilibrium states of a quasi-neutral plasma with infinite conductivity in the approximation of very small electron mass are governed in convenient units by the following set of equations:

$$
\begin{gathered}
\nabla \cdot\left(\rho \mathbf{v}_{i}\right)=0, \\
\rho\left(\mathbf{v}_{i} \cdot \nabla\right) \mathbf{v}_{i}=\mathbf{j} \times \mathbf{B}-\nabla P, \\
\nabla \times \mathbf{E}=0, \\
\nabla \times \mathbf{B}=\mathbf{j}, \\
\nabla \cdot \mathbf{B}=0, \\
\mathbf{E}+\mathbf{v}_{i} \times \mathbf{B}=\frac{h}{\rho}\left(\mathbf{j} \times \mathbf{B}-\nabla P_{e}\right)
\end{gathered}
$$

An energy equation equation or equation of state

Here, $\rho=M n$ is the ion-fluid density; $h \equiv M / e ; P=P_{i}+P_{e}$ is the total thermal pressure; the rest of notation is standard. The momentum equation (3) is derived by a superposition of the electron and ion momentum equations neglecting the electron convective velocity term because of the very small

electron mass. To make further treatment convenient Ohm's law (7) has 
been expressed in terms of mass density $\rho$ because, with the single exception of the electron pressure gradient term in (7), the set of equations (2)-(7) becomes MHD-like in form. The MHD equations are recovered in the limit of $h \rightarrow 0$. Henceforth, to simplify notation the subscript $i$ will be skipped from the ion velocity $\left(\mathbf{v}_{i} \equiv \mathbf{v}\right)$.

In this section we examine certain characteristics of HMHD equilibria in arbitrary geometry in connection with the question: under which conditions solutions of the MHD equilibrium equations can also satisfy the HMHD ones. Depending on whether the velocity term is kept in the momentum equation (3) quasi-static or stationary steady states will be examined on an individual basis as follows. The term quasi-static has been adopted because the linear velocity term always is kept in the Ohm's law.

\section{A. Quasi-static equilibria $[(\mathbf{v} \cdot \boldsymbol{\nabla}) \mathbf{v}=0]$}

Equation (3) then implies that the pressure is uniform on magnetic surfaces or $P=P(\psi)$ with the function $\psi$ labelling the magnetic surfaces (provided that such surfaces exist in three dimensional geometry). In the framework of the MHD model $(h \rightarrow 0),(7)$ on account of $(4)$ can be put in the form

$$
\mathbf{v} \times \mathbf{B}=\nabla \Phi,
$$

where $\Phi$ is the electrostatic potential $(\mathbf{E}=-\nabla \Phi)$. Therefore, $\mathbf{v}$ shares the same surfaces as $\mathbf{B}$ and $\mathbf{j}$ on which $\Phi$ is uniform $[\Phi=\Phi(\psi)]$. Coming to the HMHD model now we write (7) in the form

$$
\mathbf{v} \times \mathbf{B}=\nabla \Phi+h\left(\frac{\mathbf{j} \times \mathbf{B}}{\rho}-\frac{\nabla P_{e}}{\rho}\right) .
$$

Because of the Hall and $\boldsymbol{\nabla} P_{e}$ terms in (10), $\mathbf{v} \times \mathbf{B}$ can not in general be expressed as in (9). Therefore the magnetic surfaces deviate from the velocity ones. Assuming the ideal gas law for the electron and ion pressures $\left(P_{j}=\alpha \rho T_{j}, j=e, i\right)$ and that both the electron and ion temperatures are uniform on magnetic surfaces $\left[T_{e}=T(\psi)\right.$ and $\left.T_{i}=T_{i}(\psi)\right]$ the relation $P=\alpha \rho\left(T_{i}+T_{e}\right)$ implies that $\rho=\rho(\psi)$. It is noted that the assumption of uniform temperatures on surfaces is best fulfilled in hot plasmas because of the large thermal conductivity parallel to $\mathbf{B}$. Consequently, the second and third terms on the RHS of (10) can be put in the form of gradients. Therefore $\mathbf{v} \times \mathbf{B}=\boldsymbol{\nabla} g(\psi)$, where the function $g(\psi)$ can be constructed by 
means of $P(\psi), P_{e}(\psi), \rho(\psi)$ and $\Phi(\psi)$; thus (10) becomes identical in form with (9). This implies that if the electron and ion temperatures are uniform on magnetic surfaces any solution of the quasi-static MHD equations satisfies also the quasi-static HMHD ones. Note that then the flow must be incompressible $(\boldsymbol{\nabla} \cdot \mathbf{v}=0)$ as it follows from (2) on account of $\rho=\rho(\psi)$ and the coincidence of the velocity with the magnetic surfaces. In the case of low electron temperatures, viz. $T_{e} \ll T_{i}$, the $\nabla P_{e}$ term in (7) can be neglected and the above conclusion can be replaced by the following: For very small electron temperatures any solution of the quasi-static MHD equations with incompressible flows satisfies also the quasi-static HMHD ones.

\section{B. Stationary equilibria $[(\mathbf{v} \cdot \nabla) \mathbf{v} \neq 0]$}

Owing to the velocity term in (3), for generic flows the problem becomes very tough. For this reason we will consider incompressible flows parallel to the magnetic field:

$$
\mathbf{v}=\lambda \mathbf{B} .
$$

As in the quasi-static case it is additionally assumed that the electrons and ions obey the ideal gas law and their temperatures are uniform on magnetic surfaces. Then in MHD it was proved by one of the authors [13] that

$$
\mathbf{j} \times \mathbf{B}=\nabla h(\psi)
$$

and that the magnetic field modulus is uniform on magnetic surfaces:

$$
B^{2}=B^{2}(\psi)
$$

In fact Eqs. (12) and (13) follow under the milder assumption that the total temperature $T=T_{i}+T_{e}$ is uniform on MHD magnetic surfaces. These equations remain valid in HMHD because in both models the momentum equation is identical in form. For the reader's convenience derivation of (12) and (13) is given below.

From Eqs. (2), (6) and (11)it follows

$$
\mathbf{B} \cdot \boldsymbol{\nabla}(\rho \lambda)=0
$$

or

$$
\rho \lambda \equiv k(\psi)
$$


Inserting $\mathbf{v}$ from (11) to (3) yields

$$
\rho\left[\frac{1}{2} \boldsymbol{\nabla}\left(\lambda^{2} B^{2}\right)-\lambda \mathbf{B} \times(\lambda \boldsymbol{\nabla} \times \mathbf{B}+\boldsymbol{\nabla} \lambda \times \mathbf{B})\right]=(\boldsymbol{\nabla} \times \mathbf{B}) \times \mathbf{B}-\boldsymbol{\nabla} P
$$

or

$$
\rho\left[\frac{1}{2} \lambda^{2} \nabla B^{2}+\lambda(\mathbf{B} \cdot \boldsymbol{\nabla} \lambda) \mathbf{B}\right]+\nabla P=\left(1-\rho \lambda^{2}\right)(\boldsymbol{\nabla} \times \mathbf{B}) \times \mathbf{B} .
$$

With the aid of $P=a \rho T(\psi)$, the scalar product of (17) with $\mathbf{B}$ leads to

$$
\mathbf{B} \cdot \nabla\left(\frac{1}{2} \lambda^{2} B^{2}+\alpha T \log \rho\right)=0
$$

or

$$
\frac{1}{2} \lambda^{2} B^{2}+\alpha T \log \rho=\Pi(\psi)
$$

Owing to incompressibility, (2) implies that $\rho=\rho(\psi)$. (In fact if any of the relations $\boldsymbol{\nabla} \cdot \mathbf{v}=0, \lambda=\lambda(\psi)$ or $\rho=\rho(\psi)$ holds the other two follow.) Then (13) follows from (19) and (12) from (17).

Eq. (12), formally equivalent to the equation for quasi-static equilibria, combined with the $\psi$ dependence of $B^{2}$ has only one solution: the Palumbo solution [14]. Also, on account of $\rho=\rho(\psi),(12)$ and $P_{e}=P_{e}(\psi)$ the RHS of (10) can be expressed as a gradient. Therefore we can conclude that for magnetic-field-aligned incompressible flows and uniform electron and ion temperatures on magnetic surfaces the only solution of the HMHD equilibrium equations is the Palumbo one. Note that both assumptions $T_{i}=T_{i}(\psi)$ and $T_{e}=T_{e}(\psi)$ are required in addition to incompressibility because of the $\nabla P_{e}$ term on the RHS of (10).

\section{Axisymmetric equations}

The aim of this section is to derive reduced equilibrium equations for axisymmetric plasmas for either barotropic ion pressures or incompressible ion flows and uniform electron temperatures on magnetic surfaces. To this end certain integrals will first be identified in the form of conserved quantities either on magnetic surfaces or on ion velocity surfaces. The reduction is achieved by projecting Eqs. (3) and (7) onto three independent directions with respect to the velocity surfaces and magnetic surfaces, respectively. It should be noted here that the main derivations to follow in this Section are 
along the same lines to those in Ref. [1]. However, since Ref. [1] differs from our study in neglecting the electron pressure gradient term in Ohm's law and in adopting an isentropic ion equation of state, for the reader's convenience derivations will be given in a self-contained way here.

On account of axisymmetry and Ampere's law (5) the divergence-free fields, i.e. the magnetic field $\mathbf{B}$, the current density $\mathbf{j}$, and the momentum of the ion fluid element $\rho \mathbf{v}$ can be expressed in terms of the scalar functions $\psi(R, z), I(R, z), F(R, z)$ and $\Theta(R, z)$ as

$$
\begin{gathered}
\mathbf{B}=I(R, z) \boldsymbol{\nabla} \phi+\nabla \phi \times \nabla \psi(R, z), \\
\mathbf{j}=\Delta^{\star} \psi \boldsymbol{\nabla} \phi-\nabla \phi \times \nabla I(R, z), \\
\rho \mathbf{v}=\Theta(R, z) \boldsymbol{\nabla} \phi+\nabla \phi \times \nabla F(R, z) .
\end{gathered}
$$

Here, $(R, z, \phi)$ are cylindrical coordinates with $z$ corresponding to the axis of symmetry; the functions $\psi$ and $F$ label the magnetic and ion velocity surfaces, respectively; $\Delta^{\star}=R^{2} \boldsymbol{\nabla} \cdot\left(\nabla / R^{2}\right)$. The electron velocity lies on magnetic surfaces as it follows from the electron momentum equation with the $\left(\mathbf{v}_{e} \cdot \boldsymbol{\nabla}\right) \mathbf{v}_{e}$ term being neglected. First we will project Ohm's law (7) along $\mathbf{B}$, the symmetry direction $\boldsymbol{\nabla} \phi$ and perpendicular to magnetic surfaces $\boldsymbol{\nabla} \psi$. Using $\mathbf{E}=-\nabla \Phi(R, z)$ and $P_{e}=\alpha \rho T_{e}(\psi)$ the component of (7) along $\mathbf{B}$ yields

$$
\mathbf{B} \cdot \boldsymbol{\nabla}\left(\Phi-h \alpha T_{e} \log \rho\right)=0
$$

and therefore the quantity $\Phi-h \alpha T_{e} \log \rho$ is uniform on magnetic surfaces:

$$
\Phi-h \alpha T_{e} \log \rho \equiv \Xi(\psi) .
$$

Eq. (7) then becomes

$$
\mathbf{v} \times \mathbf{B}=\frac{h}{\rho} \mathbf{j} \times \mathbf{B}+\frac{d \Xi}{d \psi} \nabla \psi-h \alpha \frac{d T_{e}}{d \psi}(1-\log \rho) \nabla \psi .
$$

Projecting (25) along $\boldsymbol{\nabla} \phi$ yields another integral:

$$
F+h I \equiv f(\psi)
$$

Eq. (26) is consistent with the fact that the velocity surfaces depart from magnetic surfaces. The coincidence of the magnetic surfaces with the velocity 
surfaces in MHD is recovered for $h \rightarrow 0$. Furthermore, the component of (25) along $\boldsymbol{\nabla} \psi$ leads to the equation

$$
h \Delta^{\star} \psi=\rho R^{2}\left[\frac{d \Xi}{d \psi}-h \alpha \frac{d T_{e}}{d \psi}(1-\log \rho)\right]+\Theta-\frac{d f}{d \psi} I .
$$

Further reduction requires an energy equation or an equation of state for the ions. In the present study we will consider either barotropic ions, or incompressible ion flows as follows.

\section{A. Barotropic ions $\left[P_{i}=P_{i}(\rho)\right]$}

In addition to plasmas of astrophysical concern this equation of state is of relevance to self-organized states of tokamaks in connection with the so called principle of profile consistency [16]. Accordingly, the plasma is selforganized in such a way that the temperature profiles evolve in dependance with the density ones, $T=T(\rho)$, and therefore $P=P(\rho)$.

Since here $P_{i}=P_{i}(\rho)$, it is convenient to define the function $H_{i}(\rho)$ by the relation

$$
\frac{\nabla P_{i}(\rho)}{\rho}=\nabla H_{i}(\rho) .
$$

Also, we introduce the generalized vorticity

$$
\mathbf{\Omega} \equiv \mathbf{B}+h \boldsymbol{\nabla} \times \mathbf{v}
$$

The quantity $\Omega$ is divergence free and therefore it can be expressed by

$$
\boldsymbol{\Omega}=N(r, z) \boldsymbol{\nabla} \phi+\nabla \phi \times \nabla G(R, z) .
$$

Using (28), (29), $P=P_{e}+P_{i}, \mathbf{E}=-\nabla \Phi$ and the identity

$$
(\mathbf{v} \cdot \nabla) \mathbf{v}=\nabla \frac{v^{2}}{2}-\mathbf{v} \times \nabla \times \mathbf{v}
$$

Eq. (3) can be cast in the concise form

$$
\nabla W=\mathbf{v} \times \Omega
$$

where

$$
W \equiv h \frac{v^{2}}{2}+h H_{i}+\Phi
$$


Eq. (31) implies that $\mathbf{v}$ and $\boldsymbol{\Omega}$ share the same surfaces on which $W$ remains uniform or because of (22) and (30) it follows

$$
\begin{gathered}
W=W(G), \\
F=F(G) .
\end{gathered}
$$

The component of (31) perpendicular to the generalized vorticity surfaces, viz. along $\nabla G$, yields

$$
R^{2} \rho \frac{d W}{d G}=N \frac{d F}{d G}-\Theta
$$

Substituting the expressions for $\mathbf{B}$ and $\mathbf{v}$ from (20) and (22) into (30) leads to the following expressions for $N$ and $G$ :

$$
\begin{gathered}
N=I+h \boldsymbol{\nabla}\left(\frac{1}{\rho} \frac{d F}{d G}\right) \cdot \nabla G+h \frac{1}{\rho} \frac{d F}{d G} \Delta^{\star} G, \\
G=\psi-h \frac{\Theta}{\rho} .
\end{gathered}
$$

Inserting

$$
\Theta=\frac{\rho}{h}(\psi-G)
$$

$N$ from (36) and

$$
\left.I=\frac{1}{h}(f-F)\right),
$$

as it follows from (26), into (27) and (35) they become, respectively,

$$
h^{2} \Delta^{\star} \psi=h \rho R^{2}\left[\frac{d \Xi}{d \psi}-h \alpha \frac{d T_{e}}{d \psi}(1-\log \rho)\right]+\rho(\psi-G)-\frac{d f}{d \psi}[f(\psi)-F(G)]
$$

and

$h^{2} \frac{1}{\rho}\left(\frac{d F}{d G}\right)^{2} \Delta^{\star} G+h^{2} \frac{d F}{d G} \nabla\left(\frac{1}{\rho} \frac{d F}{d G}\right) \cdot \nabla G=h R^{2} \rho \frac{d W}{d G}+\rho(\psi-G)-\frac{d F}{d G}[f(\psi)-F(G)]$.

Summarizing this subsection, the axisymmetric HMHD steady states for isobaric ions are governed by the coupled differential equations (40) for the poloidal magnetic flux function $\psi$ and (41) for the generalized vorticity function $G$ together with the algebraic equations (24) and (32). The aforementioned 
equations contain the flux functions $\Xi(\psi), T_{e}(\psi), f(\psi), W(G), F(G)$ and the function $\rho(R, z)$. In $(32) v^{2}(R, z)$ and $\Phi(R, z)$ should be expressed as

$$
v^{2}=\left(\frac{\psi-G}{h R}\right)^{2}+\left(\frac{d F}{d G} \frac{|\nabla G|}{R \rho}\right)^{2},
$$

as it follows from (22) (34) and (37), and

$$
\Phi=h \alpha T_{e}(\psi) \log \rho+\Xi(\psi)
$$

by (24). The function $H_{i}(\rho)$ relates to the ion pressure by

$$
H_{i}=\int \frac{d P_{i}(\rho)}{\rho},
$$

as it follows from (28). The functional dependence of $\Xi(\psi), T_{e}(\psi), f(\psi)$, $W(G), F(G)$, and $P_{i}(\rho)$ remain free. Note that, depending on the magnitude of the poloidal velocity, Eq. (41) can become either elliptic or hyperbolic. In the elliptic regimes, once the above mentioned free functions are assigned Eqs. (40), (41) and (32) can be solved for $\psi, G$ and $\rho$ under appropriate boundary conditions. Because of the departure of the velocity surfaces from the magnetic surfaces the problem is tougher than in MHD in which the equilibrium reduces to a single differential equation for $\psi$ in conjunction with an algebraic equation for the density (see for example Ref. [15]).

\section{B. Incompressible ion flow $(\boldsymbol{\nabla} \cdot \mathbf{v}=0)$}

Some of the derivations in the previous part of this Section associated with uniform electron temperature on magnetic surfaces is unaffected and therefore Eqs. (24), (26) and (27) remain valid for incompressible ion flow. On account of incompressibility, (2) implies that the density is an ion surface quantity:

$$
\rho=\rho(F) .
$$

Using (7) and the generalized vorticity (29), the momentum equation (3) is put in the form

$$
\rho \nabla \tilde{W}=\rho \mathbf{v} \times \boldsymbol{\Omega}-h \boldsymbol{\nabla} P_{i},
$$

where

$$
\begin{aligned}
\tilde{W} & \equiv h \frac{v^{2}}{2}+\Phi \\
& =h \frac{v^{2}}{2}+\Xi(\psi)+h \alpha T_{e}(\psi) \log \rho(F)
\end{aligned}
$$


The components of (46) along $\boldsymbol{\nabla} \phi, \boldsymbol{\Omega}$, and $\boldsymbol{\nabla} G$, respectively, yield

$$
\begin{gathered}
F=F(G), \\
h P_{i}=h P_{i s}(G)-\rho \tilde{W},
\end{gathered}
$$

and

$$
\begin{array}{r}
h \frac{\left(F^{\prime}\right)^{2}}{\rho} \Delta^{\star} G+\frac{1}{2} h\left(\frac{\left(F^{\prime}\right)^{2}}{\rho}\right)^{\prime}|\nabla G|^{2}= \\
R^{2}\left[h P_{i s}^{\prime}-\rho^{\prime}\left(\Xi(\psi)+h \alpha T_{e}(\psi) \log \rho\right)\right]+\Theta-h \frac{\rho^{\prime} \Theta^{2}}{2 \rho^{2}}-F^{\prime} I,
\end{array}
$$

where the prime denotes derivative with respect to $G$. For vanishing flow the surface quantity $P_{i s}(G)$ in $(49)$ coincides with the ion pressure. Note that we have chosen the function $G$ to label the ion velocity surfaces in place of $F$. The functions $I$ and $\Theta$ in (50) can be expressed in terms of surface quantities via (26) and (37) to yield

$$
\begin{array}{r}
h^{2} \frac{\left(F^{\prime}\right)^{2}}{\rho} \Delta^{\star} G+\frac{1}{2} h^{2}\left(\frac{\left(F^{\prime}\right)^{2}}{\rho}\right)^{\prime}|\nabla G|^{2}= \\
R^{2}\left[h^{2} P_{i s}^{\prime}-h \rho^{\prime}\left(\Xi(\psi)+h \alpha T_{e}(\psi) \log \rho\right)\right] \\
+\rho(\psi-G)-\frac{\rho^{\prime}}{2} \rho^{2}(\psi-G)^{2}-F^{\prime}[f(\psi)-F(G)] .
\end{array}
$$

Recapitulating, the incompressible equilibria obey the coupled elliptic differential equations (40) for $\psi$ and (51) for $G$. They should be solved under appropriate boundary conditions for the functions $\psi$ and $G$, after assigning the free surface functions $\Xi(\psi), T_{e}(\psi), f(\psi), \rho(G), F(G)$, and $P_{s i}(G)$ they contain. The algebraic equations (49), (47) and (43) can then determine $\Phi$, $W$ and $P_{i}$.

The non-linear term $|\nabla G|^{2}$ in (51) can be eliminated by the transformation

$$
U(G)=\int_{0}^{G} M(g) d g
$$

where

$$
M \equiv \frac{d F / d G}{\sqrt{\rho}}
$$


The transformation satisfies the relations

$$
\frac{d U}{d G}=M, \frac{d G}{d U}=M^{-1}
$$

and the equation for the transformed function $U$ is

$$
\begin{aligned}
h^{2} \Delta^{\star} U= & R^{2}\left[h^{2} \frac{d P_{i s}}{d U}-h \frac{d \rho}{d U}\left(\Xi(\psi)+h \alpha T_{e}(\psi) \log \rho\right)\right] \\
& +\frac{\rho}{M(U)}\left(\psi-G(U)-\frac{1}{2} \frac{d \rho}{d U} \rho^{2}(\psi-G(U))^{2}\right. \\
& -\sqrt{\rho}(f(\psi)-F(U)),
\end{aligned}
$$

The free functions of $U$ to be prescribed in (40) and (55) are $P_{i s}(U), \rho(U)$ and $M(U)$. The functions $G(U)$ and $F(U)$ then are given by

$$
G(U)=\int_{0}^{U} M^{-1}(g) d g
$$

and

$$
F(U)=\int_{0}^{U} \sqrt{\rho(g)} d g .
$$

\section{Side conditioned axisymmetric equilibria}

In this section we will examine general characteristics of side conditioned equilibria, i.e. steady states of constant density, flows parallel to B, Beltramilike flows, purely toroidal barotropic flows, and purely poloidal incompressible flows. As will be shown, in certain cases the side conditions replace the equation(s) of state or result to equations non recoverable from the reduced ones derived in Sec. III, thus yielding different branches of equilibria. For this reason we will employ in these cases directly the original set of equations.

\section{A. Equilibria with constant density}

For $\rho=\rho_{0}=$ constant the incompressible differential equations (40) and (51) are written in the simpler forms:

$h^{2} \Delta^{\star} \psi=h \rho_{0} R^{2}\left[\frac{d \Xi}{d \psi}-h \alpha \frac{d T_{e}}{d \psi}\left(1-\log \rho_{0}\right)\right]+\rho_{0}(\psi-G)-\frac{d f}{d \psi}[f(\psi)-F(G)]$, 


$$
h^{2} \frac{\left(F^{\prime}\right)^{2}}{\rho_{0}} \Delta^{\star} G+\frac{1}{2} h^{2}\left(\frac{\left(F^{\prime}\right)^{2}}{\rho_{0}}\right)^{\prime}|\nabla G|^{2}=h^{2} R^{2} P_{i s}^{\prime}+\rho_{0}(\psi-G)-\frac{d F}{d G}(f-F) .
$$

Eqs. (56) and (57), being coupled only through the terms $\psi-G$ and $f(\psi)-F(G)$, can be decoupled by the ansatz

$$
f=\sqrt{\rho_{0}} \psi, F=\sqrt{\rho_{0}} G .
$$

The functions $I$ and $\Theta$ associated with the toroidal components of the magnetic field and the velocity then become equal each other:

$$
I=\Theta=\frac{1}{h}(f(\psi)-F(G))=\frac{\sqrt{\rho_{0}}}{h}(\psi-G) .
$$

Also, the second of (58) implies poloidal flows such that

$$
\frac{(d F / d G)^{2}}{\rho_{0}} \equiv M_{0}^{2}=1
$$

and therefore the $|\nabla G|^{2}$-term in (57) vanishes. This is the reason we have not employed transformation (52). The decoupled equations read

$$
h^{2} \Delta^{\star} \psi=h \rho_{0} R^{2}\left[\frac{d \Xi}{d \psi}-h \alpha \frac{d T_{e}}{d \psi}\left(1-\log \rho_{0}\right)\right]
$$

and

$$
\Delta^{\star} G=R^{2} \frac{d P_{i s}}{d G} .
$$

Eqs. (60) and 61) are similar in form as the Grad-Shafranov equation for $\beta_{p}=1$ equilibria. Being decoupled, however, they can be solved independently and therefore the magnetic surfaces can have different characteristics than the velocity surfaces. Linearized forms of Eqs. (60) and (61) admit a variety of analytic solutions for magnetically confined plasmas. Let us mention here in connection with known solutions of the Grad-Shafranov equation widely employed for tokamak studies the Solovév solution $[22,23]$ and the Hernegger-Maschke one [24, 25] associated, respectively, with linear and quadratic assignments of the free flux functions.

\section{B. Flows parallel to $\mathbf{B}$}


These flows satisfy the relation

$$
\rho \mathbf{v}=k(R, z) \mathbf{B}
$$

Irrespective of equation of state the component of (7) along $\nabla \phi$ yields the integral

$$
I=I(\psi) .
$$

Note that within the framework of MHD, (63) does not hold for parallel compressible flows. Also, acting the operator of divergence to (62), implies on account of (2) that the function $k$ is uniform on magnetic surfaces:

$$
k=k(\psi)
$$

Projecting (3) along $\boldsymbol{\nabla} \phi$ leads to

$$
\mathbf{B} \cdot \boldsymbol{\nabla}\left[I\left(1-\frac{k^{2}}{\rho}\right)\right]=0 .
$$

If the magnetic field is not purely poloidal $(I \neq 0)$, (65) implies that the quantity $\left(1-k^{2} / \rho\right) I$ is also uniform on magnetic surfaces and consequently

$$
\rho=\rho(\psi)
$$

Therefore, the flow must be incompressible. An alternative proof of this statement is given in Appendix.

The components of (7) along $\mathbf{B}$ and $\boldsymbol{\nabla} \psi$, yield

$$
\begin{gathered}
P_{e}=P_{e s}(\psi)+\frac{\rho}{h} \Phi(R, z), \\
\Delta^{\star} \psi+R^{2}\left(P_{e s}^{\prime}+\frac{\Phi}{h} \rho^{\prime}\right)+I I^{\prime}=0 .
\end{gathered}
$$

where $P_{e s}(\psi)$ is a "constant of integration" flux function. Furthermore, the projections of (3) along $\mathbf{B}$ and $\nabla \psi$ furnish

$$
\begin{gathered}
P=P_{s}(\psi)-\frac{k^{2} B^{2}}{2 \rho}, \\
\left(1-M_{A}^{2}\right) \Delta^{\star} \psi-\frac{1}{2}\left(M_{A}^{2}\right)^{\prime}|\nabla \psi|^{2}+R^{2} P_{s}^{\prime}+I I^{\prime}=0,
\end{gathered}
$$


where $M_{A}^{2}$ is the square of the Mach function with respect to the Alfvén velocity:

$$
M_{A}^{2}=\left(\frac{v}{v_{A}}\right)^{2}=\frac{k^{2}}{\rho} .
$$

This should not be confused with the quantity $M$ defined by (53) which is not an Alfvén Mach function. Eq. (70) is identical in form with the respective MHD equilibrium equation [17, 18]. Also, unlike the non conditioned equations (40) and (55), Eq. (70) has the Alfvén singularity $\left(M_{A}=1\right)$; therefore, parallel flows consists a different class of equlibria. The fact that the Hall effect, appearing as a non-linear singular perturbation, can remove the MHD Alfvén singularity was analyzed in Ref. [19]. Under the transformation $[20,21]$

$$
U(\psi)=\int_{0}^{\psi}\left(1-M(g)^{2}\right)^{1 / 2} d g
$$

(70) becomes identical in form with the Grad-Shafranov equation:

$$
\Delta^{\star} U+R^{2} \frac{d P_{s}}{d U}+I \frac{d I}{d U}=0 .
$$

By means of any solution of (50) Eqs. (43), (67) and (69), respectively, determine the electrostatic potential, $\Phi(R, z)$, the electron pressure, $P_{e}(R, z)$, and the total pressure $P(R, z)$.

Let us consider further the physically plausible case of uniform electron temperature on magnetic surfaces: $T_{e}=T_{e}(\psi)$. Eq. (43) then implies $\Phi=$ $\Phi(\psi)$ and consequently the differential equations (68) and (70) for $\psi$ can be cast in the forms

$$
\begin{aligned}
\Delta^{\star} \psi & =-f(\psi)-R^{2} g(\psi), \\
|\nabla \psi|^{2} & =2\left[i(\psi)+R^{2} j(\psi)\right] .
\end{aligned}
$$

Here $f, g, i$ and $j$ are known functions of $\Phi, \rho, P_{e s}, P_{s}$ and $I$. The forms of Eqs. (71) and (72) indicate that the magnetic surfaces are identical in shape with those of the Palumbo solution ( $\psi$ contours of this solution are provided in Figures 1 and 2 of Ref. [26]). The pressure, however, is not uniform on magnetic surfaces and therefore the equilibrium is not isodynamic. Only under the additional assumption $P=P(\psi),(69)$ implies $B^{2}=B^{2}(\psi)$ in accordance with the results of Section II.

\section{Beltrami-like flows}


They are defined by the side condition

$$
\mathbf{v}=\nu(R, z) \boldsymbol{\nabla} \times \mathbf{v}
$$

Eq. (3) then becomes

$$
\rho \nabla\left(\frac{v^{2}}{2}\right)=\mathbf{j} \times \mathbf{B}-\nabla P .
$$

None equation of state will be assumed either for electrons or for ions from the beginning. The $\boldsymbol{\nabla} \phi$-component of (74) implies that $I=I(\psi)$ and consequently it follows from the $\boldsymbol{\nabla} \phi$-component of (7) that $F=F(\psi)$. Therefore, the magnetic surfaces coincide with the velocity surfaces. From the $\boldsymbol{\nabla} \psi$ component of (73) it follows that $\Theta / \rho$ is uniform on magnetic surfaces:

$$
\frac{\Theta}{\rho} \equiv Z(\psi)
$$

Projecting then (73) along $\boldsymbol{\nabla} \phi$ and $\mathbf{B}$, respectively, yield

$$
\nu\left[\frac{F^{\prime}}{\rho} \Delta^{\star} \psi+\nabla\left(\frac{F^{\prime}}{\rho}\right) \cdot \nabla \psi\right]=Z(\psi)
$$

and

$$
\frac{F^{\prime}}{\rho}+\nu Z^{\prime}=0 .
$$

Note that (75)-77) coming from the side condition (73) are additional equations to those associated with the momentum equation and Ohm's law. If $\nu=\nu(\psi)$, Eq. (77) implies $\rho=\rho(\psi)$ and vice versa and therefore in this case the flow is incompressible. This case will further be considered here. The components of (74) and (7) along $\mathbf{B}$ and $\boldsymbol{\nabla} \psi$ then yield the equations:

$$
\begin{gathered}
P=P_{s}(\psi)-\rho \frac{v^{2}}{2} \\
\Delta^{\star} \psi+R^{2} P_{s}^{\prime}+I I^{\prime}-\rho^{\prime}\left(Z^{2}+\frac{\left(F^{\prime}\right)^{2}}{\rho}|\nabla \psi|^{2}\right)=0, \\
h P_{e}(R, z)-\rho \Phi(R, z) \equiv \tilde{\Phi}(\psi), \\
h \Delta^{\star} \psi+R^{2}\left[\Phi(R, z) \rho^{\prime}+\tilde{\Phi}^{\prime}\right]+f I^{\prime}-\rho Z=0 .
\end{gathered}
$$


Here $\tilde{\Phi}$ and $P_{s}$ are "constants of integration" surface functions.

Eqs. (76) and (79) can be put in the forms (71) and (72). Therefore, the equilibrium is Palumbo-like. As in the case of parallel axisymmetric flows, the pressure determined by (78) is not uniform on magnetic surfaces. Also, Eqs. (80) and (81) can determine $\Phi(R, z)$ and $P_{e}(R, z)$.

D. Toroidal $\left(F^{\prime}=0\right)$ barotropic flow

Eq. (26) then implies $I=I(\psi)$ and the generalized vorticity (30) becomes

$$
\boldsymbol{\Omega}=I(\psi) \nabla \phi+\nabla \phi \times \nabla G(R, z) .
$$

Note that although the velocity surfaces coincide with the magnetic surfaces the generalized vorticity surfaces remain departed from the magnetic surfaces [Eq. (37)]. Since $\mathbf{v}=(\Theta / \rho) \boldsymbol{\nabla} \phi,(31)$ is put in the simpler form

$$
\nabla W=-\frac{\Theta}{R^{2} \rho} \nabla G
$$

with $W=W(G)$ given by (32). The $\nabla G$-component of (83) implies that the ion rotation frequency is uniform on the generalized vorticity surfaces:

$$
\omega \equiv \frac{v_{\phi}}{R}=\frac{\Theta}{R^{2} \rho}=-\frac{d W}{d G} .
$$

Eliminating $\Theta$ from this equation and (37) yields the following relation for functions labeling generalized vorticity and magnetic surfaces:

$$
G=\psi+h R^{2} \frac{d W}{d G}
$$

Also, (27) becomes

$$
h \Delta^{\star} \psi=\rho R^{2}\left[\frac{d \Xi}{d \psi}-h \alpha \frac{d T_{e}}{d \psi}(1-\log \rho)\right]-\rho R^{2} \frac{d W}{d G}-h I \frac{d I}{d \psi} .
$$

The equilibrium is determined by the single elliptic equation (86) for $\psi$ coupled with the algebraic ones (24), (32) and (85) which contain the flux functions $I(\psi), T_{e}(\psi), \Xi(\psi)$ and $W(G)$ together with $\Phi(R, z), \rho(R, z)$ and $H_{i}(\rho)$. It is recalled that $H_{i}$ relates to the ion pressure by (44). 
As an application we will consider a plasma with constant ion and electron temperatures: $T_{i}=T_{i 0}$ and $T_{e}=T_{e 0}$. Eq. (44) then yields $H_{i}=\alpha T_{i 0} \ln \rho$. From (24) and (32) eliminating $\Phi$ one obtains the following expression for the density

$$
\rho=\exp \left[\frac{\mathcal{K}}{h \alpha\left(T_{i 0}+T_{e 0}\right)}\right]
$$

with

$$
\mathcal{K} \equiv W(G)-\frac{h R^{2}}{2}\left(\frac{d W}{d G}\right)^{2}-\Xi(\psi) .
$$

Furthermore, employing the ansatz

$$
W=a G, \quad \Xi=b \psi
$$

with $a$ and $b$ constants, (86) assumes a form independent of $G$ :

$$
h \Delta^{\star} \psi=R^{2} \exp \left(\frac{h^{2} a^{2} R^{2}}{2}\right)(b-a) \exp [(a-b) \psi]-h I \frac{d I}{d \psi} .
$$

For $a=b$ (89) reduces to the force-free-like form of the Grad-Shafranov equation

$$
\Delta^{\star} \psi+I I^{\prime}=0
$$

For $I I^{\prime}=c \psi$ with $c=$ const a solution of $(90)$ is

$$
\left.\psi=R J_{1}\left[\left(c^{2}-\kappa^{2}\right)^{1 / 2} R\right)\right]\left[a_{1} \cos (\kappa z)+a_{2} \sin (\kappa z)\right],
$$

where $J_{1}$ is the first order Bessel function and $\kappa, a_{1}$ and $a_{2}$ are arbitrary constants. In this case, however, the density profile becomes hollow:

$$
\rho=\exp \left[\frac{h a^{2} R^{2}}{2 \alpha\left(T_{i 0}+T_{e 0}\right)}\right] .
$$

Note also that the first of (88) implies rigid body rotation ( $\omega=$ const.) For this reason, to construct equilibria pertinent to magnetically confined plasmas one should consider at least $a \neq b$. This task requiring numerical solutions of (89) will not be pursued further here.

E. Poloidal $(\Theta=0)$ incompressible flow 
Eq. (37) then implies that the generalized vorticity surfaces coincide with the magnetic surfaces $(G=\psi)$ and therefore $\rho=\rho(\psi)$. In addition, from (26) and (43), respectively, it follows $I=I(\psi)$ and $\Phi=\Phi(\psi)$. Note that, unlike the case of purely toroidal flows, the velocity shares the same surfaces with the generalized vorticity [Eq. (48)]. Consequently, (40) and (51) become

$$
\begin{gathered}
h \Delta^{\star} \psi=\rho(\psi) R^{2}\left[\frac{d \Xi}{d \psi}-h \alpha \frac{d T_{e}}{d \psi}(1-\log \rho(\psi))\right]+\left[\frac{d F}{d \psi}+h \frac{d I}{d \psi}\right] I, \\
h M_{A}^{2}(\psi) \Delta^{\star} \psi+\frac{1}{2} \frac{d M_{A}^{2}}{d \psi}|\nabla \psi|^{2}=R^{2}\left(h \frac{d P_{i s}}{d \psi}-\frac{d \rho}{d \psi} \Phi(\psi)\right)-I(\psi) \frac{d F}{d \psi}
\end{gathered}
$$

with $M_{A}^{2}=(d F / d \psi)^{2} / \rho$. Eqs. (92) and (93) can be cast in the forms (71) and (72). Therefore, the equilibrium is isodynamic-like with pressure varying on magnetic surfaces by (49). Note that in the framework of MHD, except for the particular case of purely poloidal magnetic field $(I=0)$, it was proved the non existence of equilibria with purely poloidal incompressible flow [17].

\section{Conclusions}

We have studied the equilibrium of a magnetically confined plasma with flow within the framework of the ideal HMHD model by including the electron pressure gradient term in Ohm's law. Depending on whether the $(\mathbf{v} \cdot \boldsymbol{\nabla}) \mathbf{v}$ ion term is neglected or kept in the momentum equation, the ion velocity surfaces coincide or depart from the magnetic surfaces, respectively. The electron velocity always shares the same surfaces with the magnetic field. For quasi-static steady states $[(\mathbf{v} \cdot \boldsymbol{\nabla}) \mathbf{v} \equiv 0]$ without geometrical symmetry, if both the electron and ion temperatures are uniform on magnetic surfaces any MHD equilibrium solution also satisfies the HMHD equations. The flow then is incompressible. For stationary steady states $[(\mathbf{v} \cdot \boldsymbol{\nabla}) \mathbf{v} \neq 0]$ parallel to the magnetic field and electron and ion temperatures uniform on magnetic surfaces the single possible equilibrium is the Palumbo one.

For axisymmetric stationary flows in general the velocity surfaces coincide with the surfaces of generalized vorticity [Eq. (29)]. We have derived reduced axisymmetric equations when the electron temperatures are uniform on magnetic surfaces and either the ions are barotropic or the ion flows are incompressible. In the former case the equilibrium is determined by a set of two coupled partial differential equations [Eqs. (40) and (41)], the one for the function $\psi$ labeling the magnetic surfaces and the other for the function 
$G$ labeling the surfaces of generalized vorticity, together with two Bernoullitype equations. For incompressible flows the density becomes uniform on velocity surfaces and the equilibrium is determined by a set of two coupled elliptic equations [Eqs. (40) and (51)].

Furthermore, we examined the following side conditioned classes of axisymmetric equilibria: (i) equilibria with constant density, (ii) flows parallel to the magnetic field, (iii) Beltrami-like flows, (iv) purely toroidal barotropic flows, and (v) purely poloidal incompressible flows. In certain of these cases the equilibrium have different properties from the respective MHD ones; specifically, in case (ii) the flows must be incompressible while no such a restriction is imposed on respective MHD flows and, in case (v) MHD equilibria are in general not possible. The magnetic surfaces of HMHD steady states of case (v) are identical in shape with those of the Palumbo equilibrium but the pressure and the magnetic field modulus varies thereon. Similar Palumbo-like steady sates can be constructed in case (ii) for uniform electron temperatures on magnetic surfaces and in case (iii) for incompressible flows. In addition, we have constructed analytic solutions in cases (i) and (ii), and for constant electron and ion temperatures in case (iv) .

Finally, it is recalled that the main objectives of the present study were to examine general characteristics of the HMHD equilibrium model in arbitrary geometry and in axisymmetric geometry by means of the reduced equations obtained. Applications on the basis of solutions to these equations to laboratory or astrophysical plasmas, in particular by examining the impact of the Hall and electron pressure gradient terms on the equilibrium properties, requires further investigations. The study can also be extended to helically symmetric steady states.

\section{Appendix: Axisymmetric field aligned flows}

We present here an alternative proof of the statement: Axisymmetric HMHD equilibria with flows parallel to $\mathbf{B}$, with $\mathbf{B}_{\phi} \neq 0$, are necessarily incompressible. On account of

$$
\mathbf{v}=\lambda \mathbf{B}
$$

and Ampere's law (5), the momentum equation (3) can be put in the form

$$
\boldsymbol{\nabla}\left(\frac{\lambda^{2} B^{2}}{2}\right)+\lambda^{2} \mathbf{j} \times \mathbf{B}-\lambda \mathbf{B} \times(\boldsymbol{\nabla} \lambda \times \mathbf{B})=\frac{h}{\rho}\left(\mathbf{j} \times \mathbf{B}-\nabla P_{e}\right) .
$$


For axisymmetric equilibria the toroidal component of Ohm's law (7) leads to

$$
\nabla \phi \cdot \mathbf{j} \times \mathbf{B}=0 .
$$

On account of (96), projection of (95) along $\boldsymbol{\nabla} \phi$ yields

$$
\lambda(\mathbf{B} \cdot \boldsymbol{\nabla} \lambda)(\boldsymbol{\nabla} \phi \cdot \mathbf{B})=0 .
$$

If the magnetic field is not purely poloidal, (97) implies $\mathbf{B} \cdot \boldsymbol{\nabla} \lambda=0$. Consequently, from the divergence of (94) it follows $\boldsymbol{\nabla} \cdot \mathbf{v}=0$.

\section{Acknowledgements}

The authors would like to thank the Reviewer for constructive critical comments.

Part of this work was conducted during a visit of the author G.N.T. to the Max-Planck-Institut für Plasmaphysik, Garching. The hospitality of that Institute is greatly appreciated.

The present work was performed under the Contract of Association ERB 5005 CT 990100 between the European Atomic Energy Community and the Hellenic Republic. The views and opinions expressed herein do not necessarily reflect those of the European Commission. 


\section{References}

[1] V. I. Ilgisonis, Plasma Phys. Control. Fusion 43, 1255 (2001).

[2] S. M. Mahajan, Z. Yoshida, Phys. Rev. Lett. 81, 4863 (1998).

[3] S. Ohsaki, N. L. Shatashvili, Z. Yoshida, and S. M. Mahajan, Astrophys. J. 570, 395 (2002).

[4] Z. Yoshida and S. M. Mahajan and S. Ohsaki Phys. Plasmas 11, 3660 (2004).

[5] A. I. Morozov, Plasma Phys. Reports, 22, 117 (2002).

[6] Z. Yoshida and S. M. Mahajan, Phys. Rev. Lett. 88, 095001 (2002).

[7] L. J. Palumbo and A.M. Platzeck, J. Plasma Physics 72, 457 (2006).

[8] A. I. Morozov and L. S. Solovév, Reviews of Plasma Physics 8, 1 (1980).

[9] K. Avinash, Phys. Fluids B 4, 3856 (1992).

[10] L. C. Steinhauer, Phys. Plasmas 62734 (1999).

[11] H. Yamada, T. Katano, K. Kanai, A. Ishids, L. C. Steinhauer, Phys. Plasmas 11, 4605 (2002).

[12] A. Thyagarja and K. G. McClements, Phys. Plasmas 13, 0625202 (2006).

[13] H. Tasso, Phys. Lett. A 222, 97 (1996).

[14] D. Palumbo, Nuovo Cimento B 53507 (1968).

[15] E. Hameiri, Phys. Plasmas 26230 (1983).

[16] B. Coppi, Comments Plasma Phys. Contr. Fusion 5, 261 (1980).

[17] H. Tasso, G. N. Throumoulopoulos, Phys. Plasmas 8, 2378 (1998).

[18] G. N. Throumoulopoulos, G. Poulipoulis, G. Pantis, H. Tasso, Phys. Lett. A 317, 463 (2003).

[19] Jun-ya Shiraishi, S. Ohsaki, and Z. Yoshida, Phys. Plasmas 12, 092308 (2005). 
[20] R. A. Clemente, Nucl. Fusion 33, 963 (1993).

[21] P. J. Morrison, private communication: this transformation was discussed in a talk entitled "A generalized energy principle" which was delivered in the Plasma Physics Division Meeting of the APS, Baltimore, 1986.

[22] V. D. Shafranov, Rev. Plasma Phys. 2, 103 (1966).

[23] L. S. Solovév, Rev. Plasma. Phys. 6, 239 (1976).

[24] F. Hernegger, in Proceedings of the 5th EPS Conference on Controlled Fusion and Plasma Physics, Grenoble, 1972, edited by E. Canobbio et al. (Commissariat a l'Energie Atomique, Grenoble, 1972), Vol. I, p. 26.

[25] E. K. Maschke, Plasma Phys. 15, 535 (1973).

[26] C. M. Bishop and J. B. Taylor, Phys. Fluids 29, 1114 (1986). 